\title{
An information centric framework for creating virtual environments to support micro surgery
}

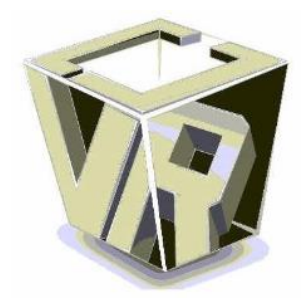

\author{
J. Cecil ${ }^{1}$, Ph.D , Miguel Pirela-Cruz ${ }^{2}$, M.D.
}

1. Associate Professor and Director, Center for Information Centric Engineering (CICE), School of Industrial Engineering and Management, Oklahoma State University, Stillwater, Oklahoma,j.cecil@okstate.edu

2. Professor \& Department Chair, Orthopaedic Surgery, Paul L. Foster School of Medicine, Texas Tech Medical Center in El Paso,Texas,Miguel.cruz@ttuhsc.edu

\begin{abstract}
In this paper we discuss the creation of an information centric framework to develop a virtual reality environment for micro surgery. An information model of micro surgery was built through interactions with an expert micro surgeon using the engineering Enterprise Modeling Language (eEML). The overall approach and architecture of the micro surgical environment is discussed. An enterprise level surgical manager, surgical planner and other components work together to enable the functioning of the virtual environment for micro surgery. Such virtual environments are essential to educating / training young budding surgeons..
\end{abstract}

Index Terms - Virtual reality, Information model, micro surgery, surgical training, information technology

\section{INTRODUCTION}

The use of Virtual Reality simulation in surgical training is gradually increasing as a result of changes in residency requirements [25], [26]. The need to develop safer, efficient environments has catalyzed the development of alternate techniques for the training of surgeons. The traditional methods run the risk of high cost and the possibility of post operative complications. Moreover, animal models are no longer an acceptable practice for teaching surgery. In this context there is a need to create virtual environments for teaching surgical and other procedures as well as for investigating new techniques for specific domains in the field of medicine. Micro surgery has been selected as the surgical field for various reasons. It has been acknowledged that the micro surgery field, in general, is in a crisis state as the number of surgeons being trained in this field has been gradually decreasing [27]. As micro surgery is considered more difficult than other surgical fields (requiring more years of training), the number of medical residents entering this field has reduced considerably. The creation of a virtual surgical environment for micro surgery will be useful in addressing this problem by accelerating and supplementing existing training experiences for surgical residents (or students).
The creation of a virtual surgical environment will enable surgical residents to learn the appropriate way of managing various conditions with safety considerations to the residents (students) and patients. It will enable to assess as well as propose alternative ways to respond to to specific medical conditions. Such virtual environments are essential to educating / training young budding surgeons. The traditional way of surgical teaching involves students first merely observing a 'live' surgery and then gradually progressing to assisting experienced surgeons [25], [26]. Medical residents currently also acquire their skills by performing surgeries on cadavers; however, there are some inherent challenges in adopting such approaches including availability, cost and the possibility of infections, which limit their usefulness. There is a need to develop virtual environments which can be used to teach surgical skills to medical residents and budding surgeons. There is also a related need to develop a strong foundation using information centric principles that will enable modeling the complex relationships within a surgical process. Such an approach would provide a strong foundation to design and build surgical environments.

In the paper, the focus of discussion is on the design of an information centric framework for Micro $\underline{\text { Surgery. }}$ Such a framework comprises the following aspects:

(a) the development of an information oriented process model (or information model) which can be used as a 'process' basis to design/build the surgical environment

(b) the use of such an information model in propelling / guiding the various surgical simulation activities

(c) the architecture and functioning of the virtual surgical environment

The scope of discussion of the simulation environment does not include implementation of the haptic interface and validation of the simulation environment. The emphasis in this paper is more on the overall design of the virtual surgical environment as well as the use of information models to propel the simulation activities. The role of information models in obtaining a robust understanding of the micro surgical processes is also discussed in this paper. This surgical environment will be useful in educating and training surgical residents intending to specialize in micro surgery. As the proposed virtual environment evolves, it can also be used as a 
research environment where surgical alternatives can be explored by practicing surgeons.

Current trends for medical / health related education include the use of simulations in a range of areas such as heart surgery, dermatologic surgery etc. [28-31]. There have been a limited number of research efforts which have explored the use of Virtual Reality technology in developing simulation environments to facilitate various procedures including surgery [25], [26].

\subsection{Understanding the complex domain of micro surgery using information oriented process models}

The virtual surgical environment was created after interacting with micro surgeons who served as experts to this complex process domain. The emphasis is on 2 aspects: (1) obtain an understanding of the temporal precedence constraints and relationships among the various tasks and sub tasks (2) create an information oriented process model (or simply an information model) that reflects the attributes based on (1). The information model serves as the basis to create Virtual Surgical Environment. The creation and content of the information model is discussed in section 3 of this paper. While several research initiatives have attempted to create virtual environments [1-19], a major drawback is the lack of emphasis is obtaining a structured understanding of the target surgical process being simulated. In our approach, we stress that this formal and structured understanding lays the foundation for a robust surgical environment which captures the complex relationships among the various entities, inputs and controlling factors coming into play during a target surgical process (figure 12).

\subsection{Propelling the simulation activities using an information model}

One of the major drawbacks of other virtual environments for medical applications is the inability to easily modify or extend the scope of the simulations for various training activities. To overcome this problem, we have proposed the use of an information model which can be interfaced with the functioning of the various modules in the virtual environment. In our approach, the subject experts or other knowledge engineers can quickly update, change or modify the behavior of the virtual environment by changing various entities in the information model. The advantage of such an approach is that by changing the information model, the simulation activities can be modified at various levels of abstraction. For example, a given surgical processes which involves a specific tool and a specific surgical path/approach can quickly be modified to demonstrate a different surgical path/approach involving a different set of tools and information inputs. We refer to this innovative approach as an information model driven simulation approach.

\subsection{Architecture and modules of the Virtual Surgical environment}

The discussion of the architecture and the various modules is limited to the progress in Phase I of this project. The long term interest is to create a robust high fidelity virtual environment that will enable a medical resident (or surgeon in training) to be assessed as well as allow the budding surgeon to propose alternative ways to surgically respond to a specific medical condition. Such virtual environments are essential to educating / training young budding surgeons and /or surgical residents.

Section 2 of this paper provides a literature review of relevant research projects relating to use of virtual environments for surgery and medicine. The design of the virtual suturing modules and the other main modules of the Virtual Surgical Environment is discussed in section 4 of this paper. Section 5 is the conclusion.

\section{REVIEW OF RELATED LITERATURE}

In this section, a brief overview of related research involving virtual reality and surgery is provided [1-23, 28-34]. The use of surgical virtual reality devices has increased over the past decade and there has been a steady evolution from basic non haptic based training tools to more advanced procedural simulators. Virtual Reality simulators have an advantage over other traditional computer based systems as they allow the users to practice and improve their skills as well as compare their performance with their peers. They provide objective assessments of the tasks performed by the user as against the traditional systems which are subjective and based on the judgment of the observer [17].

Currently, virtual reality simulators are mostly used for cardiac, dental, dermatology surgeries [25]. In the domain of dental surgery, a recently published paper discusses the design of a virtual dental surgical environment. This simulator allows dentists to diagnose and treat the periodontical diseases by interacting/visualizing a 3D 'virtual mouth' region which also provides tactile sensations using haptic technology [16].

Virtual Reality can also be used for planning surgeries. One such approach is discussed in [15] that involve liver surgery domains. In this reported project, virtual reality based tools facilitate planning approaches which includes three main stages (a) Image analysis, (b) segmentation refinement, and (c) treatment planning. The treatment planning tool is used to elaborate a detailed strategy for surgical intervention including an analysis of important quantitative indices such as volume of healthy liver tissue that will remain after surgery [15].

The analysis of the virtual reality systems revealed that training on virtual reality simulators reduced the time taken to complete a given surgical task as well as clearly distinguishing between the experienced and the novice trainees [14].

Another surgical tool is "SPRING", which is a realtime surgical simulation system that supports soft tissue modeling, and anatomy acquisition. It provides a general framework for surgical simulation that can support the requirements of many surgical simulations with an emphasis on real time performance [13].

One of the research studies involved analyzing the effect of proficiency-based VR training on the outcome of the first 10 entire cholecystectomies performed by new residents. The results showed that training on the VR simulator to a level of proficiency significantly improves intraoperative performance during a resident's first 10 
laparoscopic cholecystectomies [32]. Another report outlined the use of a 3D virtual reality system for planning minimally invasive neurosurgical procedures; the preoperative 3D model provides enhanced surgical confidence [33]. A number of studies have been conducted to demonstrate that skills acquired during VR training, transfer to the operating room [34].

There has been only a limited amount of research conducted in the area of information collection and domain understanding in the context of medical surgeries. Jalote-Parmar et al [24] discussed the role of a Work flow integration matrix (WIM) to support the design of surgical information systems. WIM uses theories of human behavior in problem solving and seeks to explore the role of evidence-based decision-making for the development of new surgical technologies. In general, there has been a lack of emphasis on using formal information models to gain a better understanding of target surgical processes. Further, the reviewed simulation environments do not have a direct or easy way to expand the scope of their capabilities nor have an easy way to modify a surgical procedure. In this paper, we emphasize addressing these two issues by using an information modeling approach (section 1.1 and 1.2 provided an overview of these principles).

\section{OVERVIEW OF THE ARCHITECTURE OF THE VIRTUAL SURGICAL ENVIRONMENT}

The architecture and main components of Virtual Surgical Environment is illustrated in figure 1. The user interaction module manages the interaction with a user (which could be a surgical resident or surgeon or others).

The overall software environment is managed by an enterprise level manager called the Surgical Scenario Manager (SSM). One of the unique attributes of is the role of information intensive process models. These process models can be used to drive the overall interactions to ensure that the simulation activities adhere to a process driven emphasis similar to process engineering activities in various engineering domains. This information model can be created using eEML (the engineering Enterprise Modeling Language) and used as the foundation to also understand the complex process of micro surgery. This information model and modeling approach is discussed in section 4 and 6 of this paper.

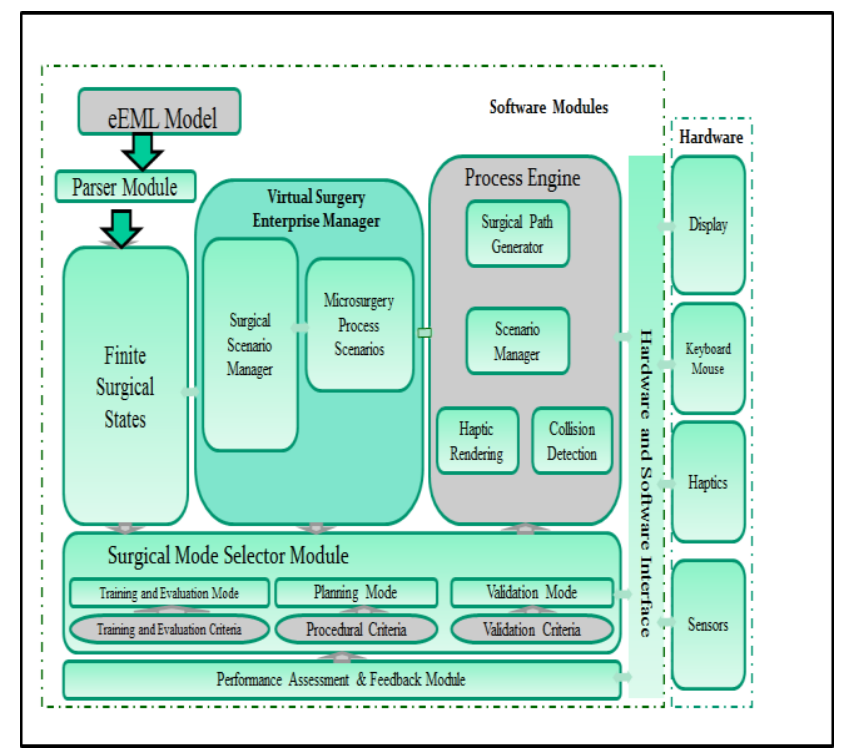

Figure 1: Architecture of Virtual Surgical Environment

The information model can be created using MS Visio and stored as an XML file. The details of the various fields (or attributes shown in figure 15, for example) can be retrieved or extracted with the help of a parser, which has been created using $\mathrm{C}++$. The functioning of this information model driven interface is discussed in section 4.

The simulation, visualization and interaction are managed by the Virtual Reality Process Engine. There are several modules managed in this engine which include the surgical path generation module, the scene management module, the haptic interface module as well as the collision detection module. The virtual reality environment was created using $\mathrm{C}++$ and Coin 3D libraries.

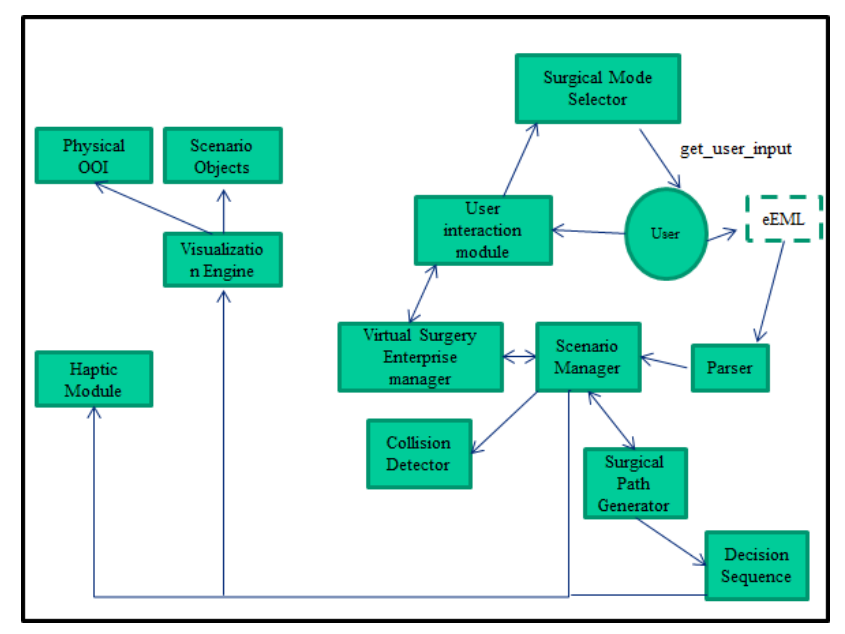

Figure 2: Interactions among the various modules of Virtual Surgical Environment

\subsection{The User Interaction Module}

Through this module, users can provide necessary information for the simulation as well as select the mode of interaction. The long term scope of Virtual Surgical Environment is to provide three options:

(a) Training and Evaluation mode

(b) Planning Mode 
(c) Validation Mode

Users can input an information model based on eEML. In phase I, the Virtual Surgery Enterprise Manager (VSEM), which is a software manager for the Virtual Surgical Environment, only provides the option for an eEML data file for a given surgical approach (this is discussed in the later sections of this paper). eEML models can be created using Visio software, which can then be exported as an XML file. This XML file contains various eEML decompositions of the tasks in a given surgery as shown in Figure 5.

Physical resources, software objects, information objects, physical objects, new information, feedback information, and updated information. The main $\mathrm{C}++$ classes used to extract this information as well as interface with other modules (Scenario Manager and VSEM) are indicated in Figure 5. The parsed information is then sent back to the Scenario Manager and the VSEM to be used as the basis for managing the simulation scenarios. The VSEM creates a decision sequence flow chart which governs the overall process to be simulated. The changes in the decision objects (DOs, see figure 16-18) highlight the key state changes within a target process being simulated. Each activity in the simulation corresponds to the E-n entity in a given eEML model. For example, for the process, 'perform the knotting procedure', the Scenario Manager reads the extracted information using the parser and then calls the appropriate functions to orchestrate the simulation. For the process shown in figure 18 (see E-5), the Scenario Manager executes the function for the knotting procedure which is the key information driver for this process. Once that simulation process (for knotting) is completed, it updates the decision sequence flow chart which stores three specific variables in each scenario: new information entity, updated information entity and physical object entity. As a knot has been simulated, the physical object entity will update its status to 'completed knot'. New information and update information entities will update their entries based on the process itself. If a user has not satisfactorily completed a knotting procedure (indicated by being not able to follow the suture trace shown in the virtual environment, see figure 20), this will be displayed

In the training mode, the surgical residents can choose a specific scenario to be trained. When a user chooses to understand a given surgical process the virtual environment provides a detailed simulation highlighting the key steps from start to finish. With the help of the planning, interaction and haptic interface modules, these residents can complete specific micro surgical procedures interactively. The planning mode is primarily responsible to allow expert surgeons to specify tasks involved in a target surgery. They can interact either through eEML models or specify lower level path plans which indicate the path of the surgical tools and other entities. The validation mode enables expert surgeons to validate the surgical plans and details proposed by the automated planner. In this paper, the emphasis is only on the training mode.

In modeling our surgical process, we initially divided the overall process into scenarios, which in turn are decomposed into activities (these in turn can be

\subsection{The Parser Module and Scenario Manager}

The purpose of the parser module (figure 3) is to extract the surgical process information from the eEML model. First, an eEML model is created in Microsoft Visio (figures 15, 16, 17, 18); subsequently, this eEML file is exported as an XML file. The parser (written in $\mathrm{C}++$ ) then reads the information attributes in the eEML model via an XML file. These attributes include constraints, information inputs, physical inputs, teams,

decomposed into other child activities depending on the user's level of detail and interest; figures 16,17 and 18 are examples of these decompositions). In figure 8 , the surgery process is indicated as the verb phrase in E-0 (which corresponds to 'Perform the suture of blood vessels') with six corresponding scenarios (E1 through E6, in figure 16-18). E1 is decomposed into other surgical activities (E1-1 through E1-4). Depending on the scenario involved, the Scenario Manager may require additional input from the user. For example, for E 5 (performing the knotting procedure), in Phase I, there is no automatic generation of the 3D suturing path. This path is input through a data file by the user. A data adaptor manages the data exchange specifications (see figure 6).

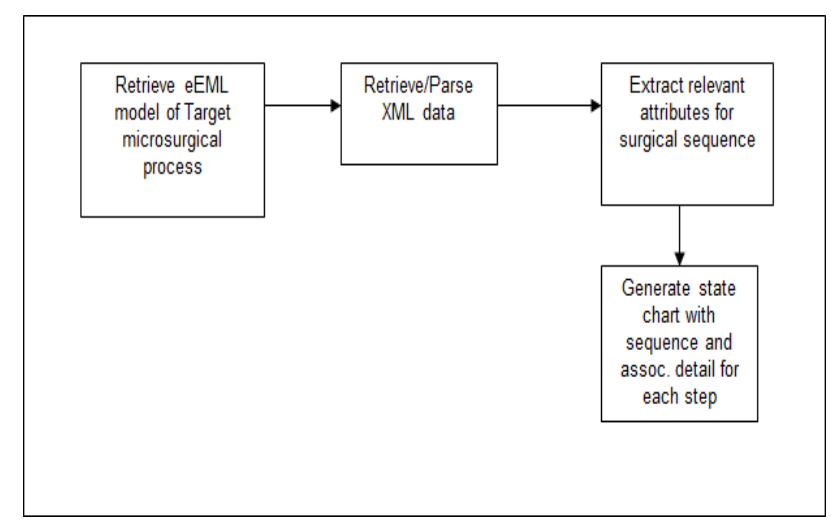

Figure 3: Using the eEML model to propel the simulation activities

The input data (or information) is necessary for path planning and simulation management. After a given eEML model has been parsed, the VSEM instructs the Scenario Manager to request the needed information (based on this model); such information drivers are identified in the information input attributes of the eEML model for that scenario. For some scenarios, the appropriate data is requested from user or from legacy data files. Examples of such legacy data can be the prestored sequence of a widely used surgical pattern or technique along with descriptions of suturing paths and other details such as a surgical cut (where parameters such as depth, width, etc can be defined). This data can be stored using XML (Extensible Mark-up Language). XML has been selected for use for a number of reasons including simplicity, openness, extensibility, self descriptions as well as separation of content from presentation. 
A typical suturing path can be a subset of a larger surgical scenario. The scope of a complete surgical scenario can be defined by a surgeon or team of surgeons in eEML. The retrieval of a pre-determined set of surgical paths can be specified as one of the children or lower level activities with corresponding association to suturing paths embedded as 'information inputs'.

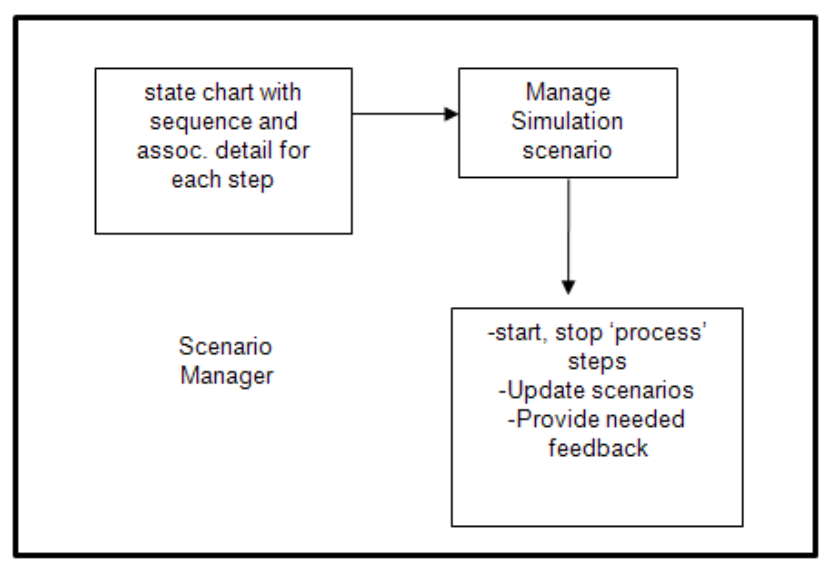

Figure 4: The Scenario Manager

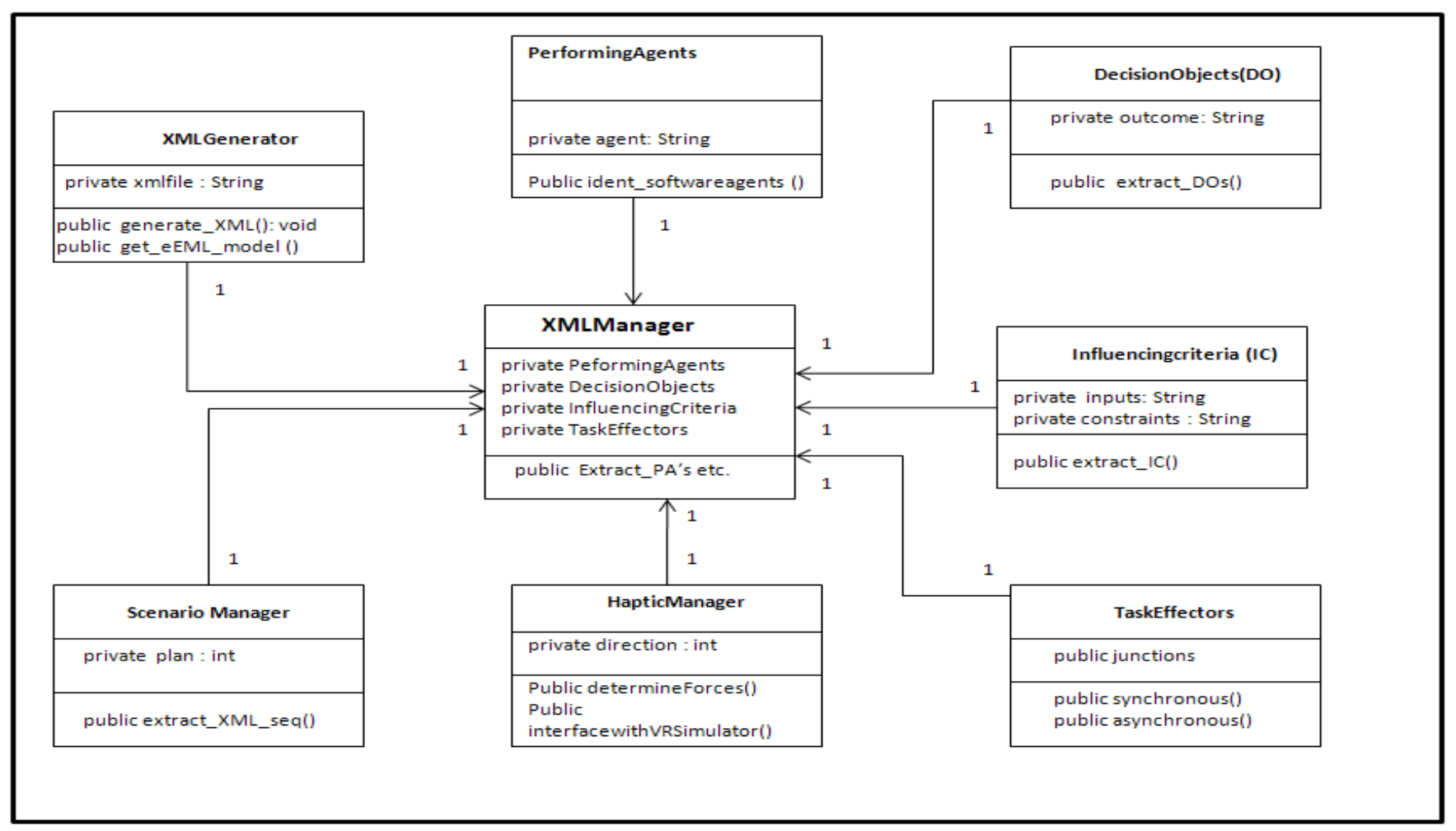

Figure 5: The main classes involved in the extraction of the eEML model

The Scenario Manager manages the Virtual Environment for target surgeries including managing interfaces with relevant display devices and environments (figures 4 and 5). The VSEM is also the software coordinator responsible for managing the homogeneous virtual environment with many simulation threads and input data from many sources. In the long term, it will also provide a mechanism for collaboration between other dedicated surgical environments for other surgical processes (such as orthopaedic surgical process, etc.).

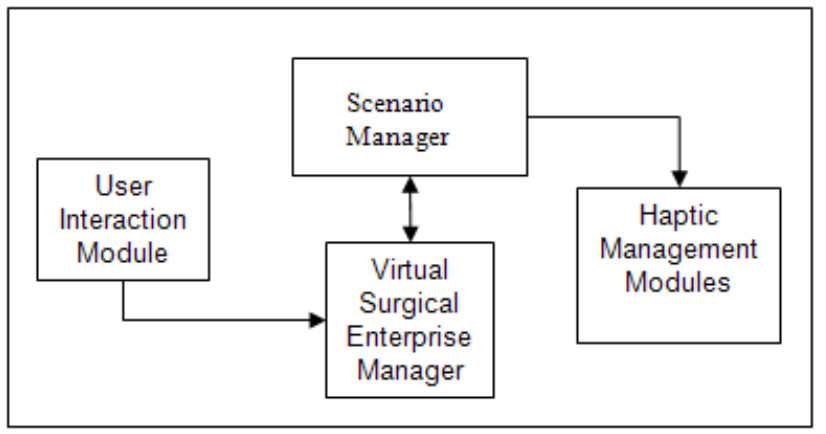

Figure 6: Interactions between Surgical Enterprise Manager, Scenario Manager and Haptic Management modules

The User Interaction Module (UIM) also contains user's profile and settings for each user including temporal and persistent information. It is also responsible for multi-user collaboration during a given surgical process or scenario. It has a list of users' profiles, settings and the mechanism and communication protocols. The Scenario Manager interacts closely with the Virtual 
Surgical Enterprise Manager and the Haptic Management modules (fig 6). Currently, the haptic management modules have not been implemented. Their design is discussed in a later section of this paper.

The Scenario manager is also responsible for providing analysis outputs after a simulation session. The Haptic Management modules interface with specific haptic devices, which will eventually be incorporated as part of the Virtual Surgical Environment.

\section{SURGICAL PLANNING AND MANAGEMENT IN THE VIRTUAL REALITY ENVIRONMENT}

In phase I, the emphasis is on the domain of micro surgeries involves veins and arteries. An expert surgeon can specify the detailed path and provide details such as the direction of the surgical needle (for example) as well as interactively specify the path to be followed in performing various surgical details. The overall approach can be illustrated using an example involving a micro surgical process that involves suturing a nerve (as seen in figure 7).

Figure 8 shows the overall decomposition for such a process. The main top level activity is E-0, which in turn is composed of 6 children activities labeled E-1 through E-6. The detailed information contained in this model becomes the basis for the simulation. By changing the various attributes of the corresponding information model (stored first in eEML and then extracted from an XML file), a range of surgical conditions and details can be simulated for planning and training purposes.

A given surgical simulation process (in our approach) is defined to be composed of corresponding process scenarios which in turn contain the detail of the actual surgery to be simulated in the form of a state chart. The eEML model provides this process context by indicating which attributes are relevant for a specific simulation. Using a unique set of modeling attributes including information / physical inputs, driving constraints, performing agents and decision outcomes, a detailed state chart can be maintained which propels the functioning of the simulation environment. The structure of the eEML models is discussed in the next section of this paper.

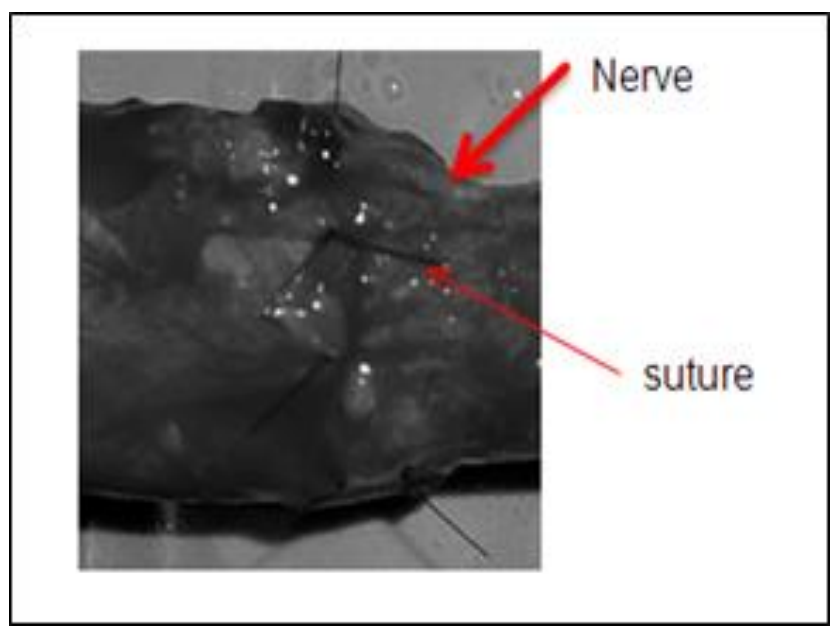

Figure 7: Suturing a nerve through micro surgery

eEML is a modeling language that can be used to model functional and process activities; other modeling languages such as IDEF-0 have been used to model complex processes such as designing fixtures for manufacturing [35]. The E0 activity (see figure 15) corresponds to the target surgical process of interest (in this case suturing of blood vessels). This process (as described earlier) is composed various scenarios (E1 through E6); the simulation of these scenarios and the interactions between the user and the simulation environment forms the basis of the training, planning and validation sessions. For the suturing process (shown in figure 7), the preliminary step involves building a corresponding eEML model or obtaining it from eEML data base (containing standard surgical processes). In our framework, each micro surgical process has a corresponding detailed eEML model which spans various levels of detail or abstraction. In the information model shown in figure 8 , the overall process E-0 is divided into 6 children activities or scenarios (E-1, E2,...E-6).

Subsequently, each of these scenarios can be further divided into other detailed child activities (see E-11, E12, etc, in Figure 5; a limited number of the corresponding eEML model decompositions in figures 16-18). The details of a target process are stored as an information scenario file (which is a Visio file). Subsequently, a corresponding XML file is generated corresponding to this scenario. This XML file contains the detailed attributes to describe the overall flow of activities for micro surgery. A parser written in $\mathrm{C}++$ extracts this information; the Scenario Manager uses these attributes to simulate the lower level process characteristics which begin with positioning the blood vessel, and continues with positioning the needle, passing the needle through the blood vessel, knotting the suture and finally performing the patency tests. The various attributes used to describe these scenarios are discussed in section 5 of this paper. The eEML provides an information rich process context which enables the creation of a detailed simulation environment.

The virtual environment is composed of various entities of interest (figure 10). The EOI's can range from physiological attributes such as veins, arteries (and other attributes corresponding to organs in a human body) as well as mechanical attributes used in surgery by the micro surgeon. These mechanical attributes can range from a blood vessel dilator to an H-clamp used during surgery. A real world view of a micro surgical environment is shown in figure 9. In figure 10, the corresponding virtual environment created is shown involving the same entities. 


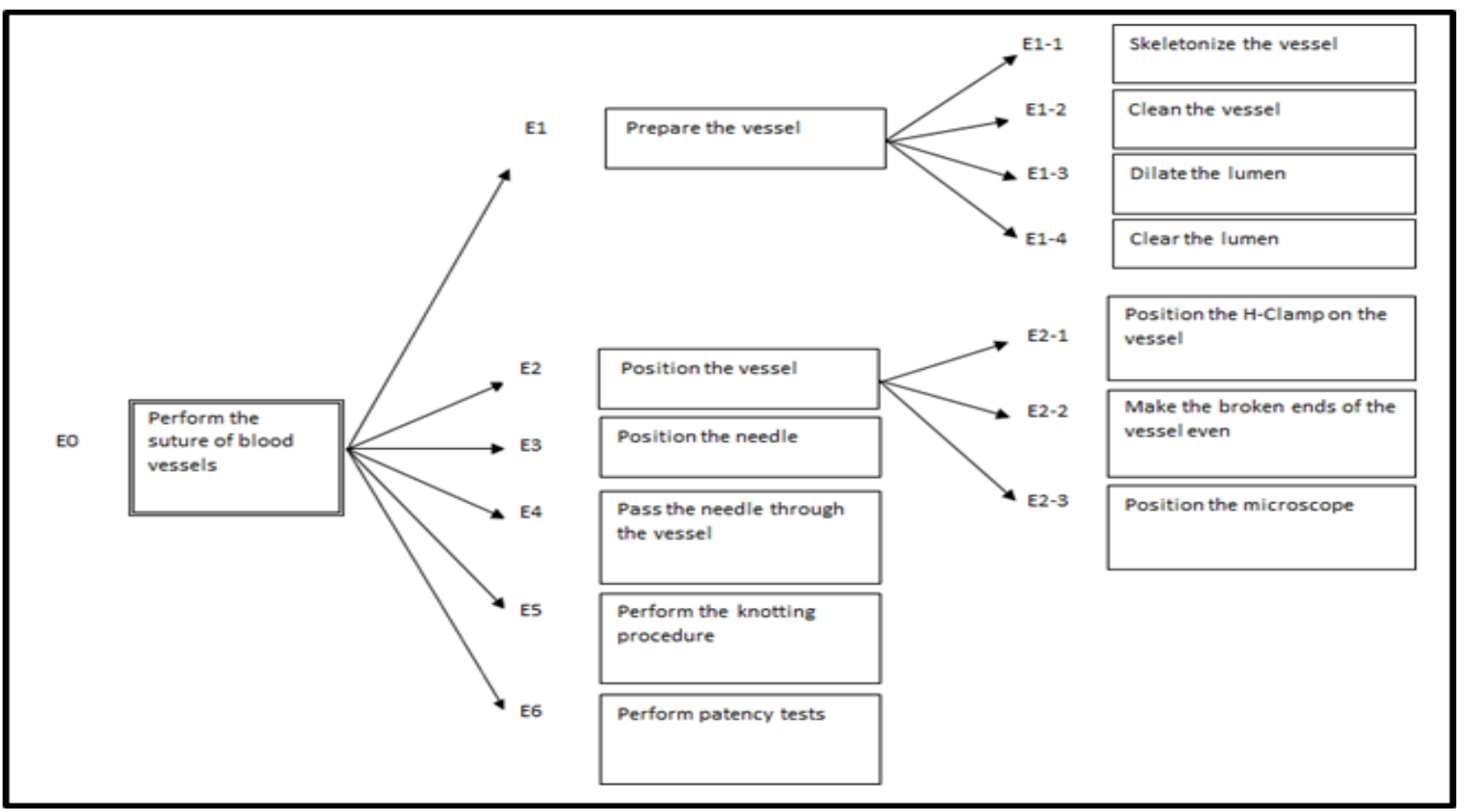

Figure 8: The decomposition of the suturing process in micro surgery

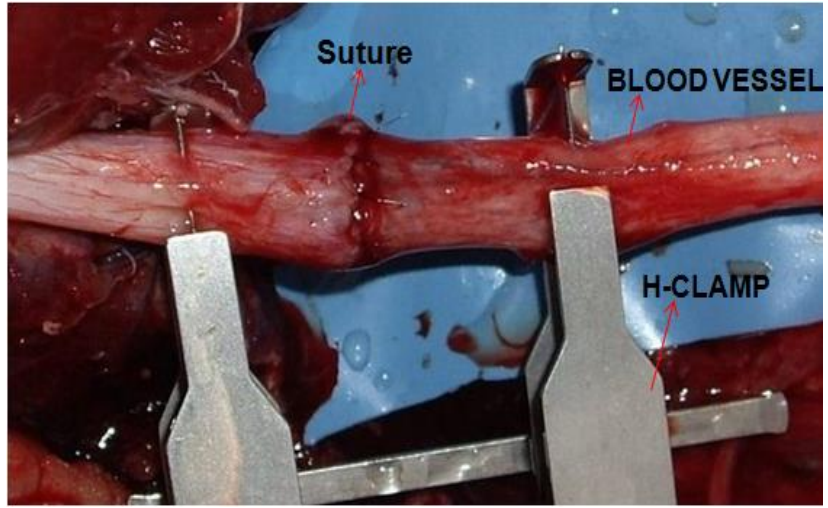

Figure 9: The mechanical and physiological entities in a typical micro surgical scenario

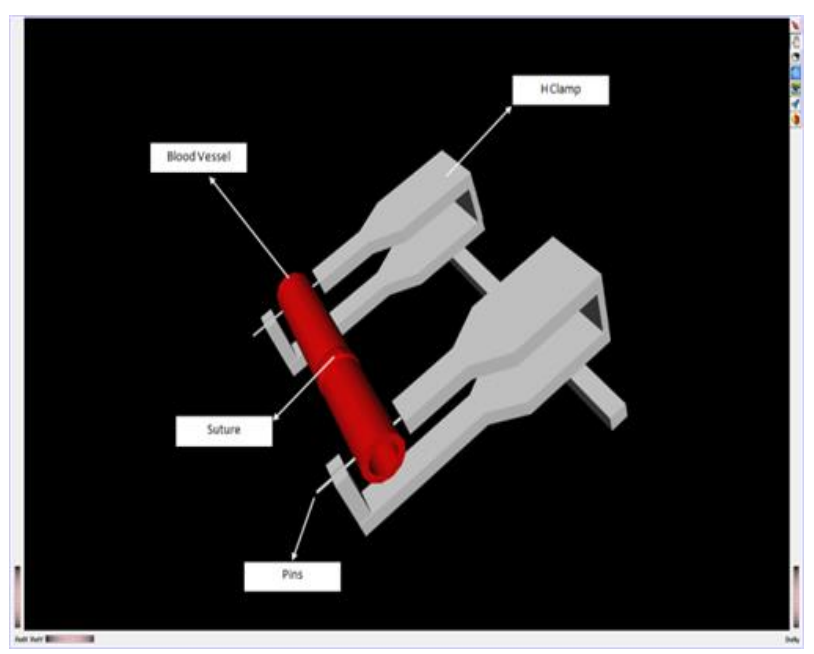

Figure 10: A view of the virtual environment for microsurgery

\section{DESIGN OF THE HAPTIC MODULE AND OTHER INTERFACES}

An overview of the preliminary design of haptic and other interfaces is provided in this section to enable a better appreciation of the overall scope of the virtual environment. We have explored the use of the Sensible Phantom haptic device to interface with the virtual environment. The Phantom is a 3 DOF physical device and 6 DOF force feedback, resolution is 3 millimeters so it can simulate many suturing scenarios with typical instruments such as needles and clamps. The friction and the mutual reaction forces need to be computed for haptic feedback during a suturing session.

The feedback approach for a typical Virtual Suturing session can begin when the user (medical intern or surgeon) moves the stylus (handle) of the haptic device. The encoder (which is a light sensor) inside the device can locates the position (pose) of the haptic device and transmit this to associated adaptor modules. These modules can receive and process such data which can be interpreted by other modules.

The Scenario Manager can then obtain this haptic information to render a scene. The haptic device can be represented in a scene as a corresponding virtual graphic object (or avatar) in the virtual environment.

The haptic avatar can assume various 3D shapes relevant to a given surgical process and scenario. For the suturing process, the shape of the needle can appear as a fishing hook (figure 13) and small as a human hair; in a micro surgery, the surgeon uses a needle holder to complete the surgical process (figure 14). The collision detection feature can be invoked when the needle starts touching the surface of a target tissue area. The collision detecting approach can be based on computing a convex hull of the two bodies which can potential come into contact. Testing for intersection of these convex hulls enables the detection of collisions. If the contacting points are not over the allowance offsets from the 
planning path, the relevant forces are computed and sent to controller to which in turn is communicated to the haptic device for generating simulated forces. However, if a user goes along an incorrect path (this is detected by the path of the virtual needle), a default vibration signal can be sent to the controller which can be used to display a warning message during the simulation. In these situations, the scene can be updated when the needle moves or after a collision event is triggered.

When using such a virtual surgical environment, two basic options can be provided to the user:

(a) The non-immersive option (fig 10) where the user can interact and be trained on a regular PC environment without being completely immersed within the virtual environment

(b) the immersive option (figure 20) which allows users to navigate using stereo vision eye wear, trackers and sensors which support complete immersion using a PowerWall system (from Mechdyne). Several modes of interaction are being explored. In the constrained mode, a user can only perform the surgical steps the system allows them to be trained in. With the help of haptic cues and constraints, the system allows only movement in the direction and path of a target scenario (for eg: the

The internal management of the various virtual entities is the responsibility of the visualization engine. The visualization engine uses a scene graph approach and is implemented using Chai $3 \mathrm{D}$ and $\mathrm{C}++$ tools. A scenario for simulation is first created using the relevant CAD models corresponding to the physiological and mechanical entities (shown in figure 10) which correspond to the ruptured vein, H-Clamp, Pins (etc.) and are imported into this scene database. The ancillary attributes are added as children to their respective group nodes. Each of these group nodes contain commands for accurate positioning and orientation of CAD models (rotate, transform and translate). Additional discussion on the implementation using Chai $3 \mathrm{D}$ is provided in section VII of this paper.

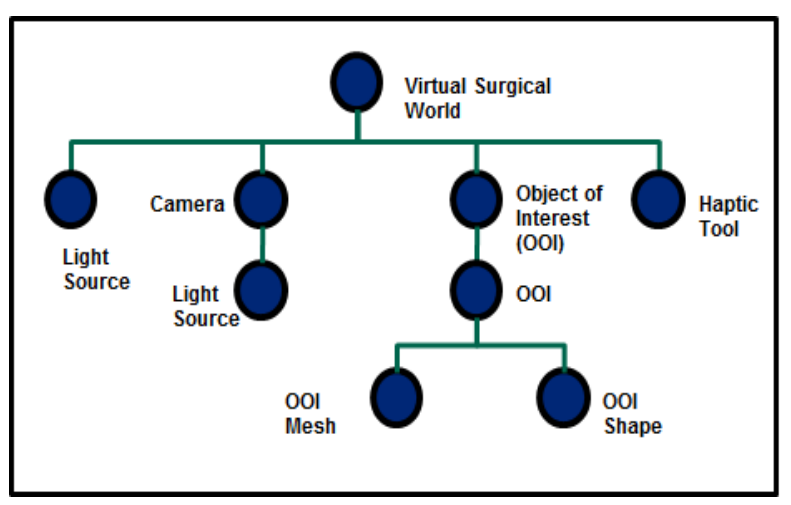

Figure 11: Internal representation of various entities in the virtual environment

\section{ROLE OF INFORMATION MODELING IN OVERALL FRAMEWORK}

The virtual reality simulators for surgery which are currently in practice have not explored the use of suturing path discussed earlier). In the unconstrained mode, a user can first propose a suturing path and then proceed to complete the suturing task of interest.

In Phase I development cycle, evaluation and feedback of a surgical resident's performance is not provided. These are addressed in the Phase II developmental cycle. The second option within training (which will be implemented in Phase II) is to select the interactive evaluation process. In this option, the user attempts to perform a process he/she has studied through an earlier simulation and then repeats this process. In the immersive option, users can navigate more effectively using motion trackers and $3 \mathrm{D}$ stereo eyewear which are linked to an advanced Power Wall system (from Mechdyne). While one surgical resident (or primary user) can interact using a controller and an active stereovision eyewear, other users can also be immersed with the primary user. This allows the medical training team to compare options, discuss strategies and communicate more effectively inside the virtual environment. The controller allows the user to zoom in/out, turn, and fly around. A directional wand in $3 \mathrm{D}$ enables the user to be aware of a general direction of movement.

information models. In our information centric framework, an unique aspect is the use of information models which can be used to propel the overall surgical simulation process.

In our approach (which reflects our framework), the distinguishing focus is on understanding the "process" of performing micro surgery. We have studied and modeled Micro surgery from a process engineering perspective with an emphasis on information centric principles. These Information modeling techniques have been used to obtain a better understanding of the micro surgical process. The various key relationships, constraints and performing mechanisms along with the temporal precedence constraints will be modeled using the engineering Enterprise Modeling Language (eEML). A key emphasis was on identifying functional relationships among various attributes underlying the process of micro surgery with a view towards using that understanding to create an advanced virtual environment to train micro surgeons. We have developed an information model based on interactions with expert surgeons (as part of our interest in obtaining an understanding of the surgical activities). A specific emphasis is also on the planning activities involved in micro surgery. While other virtual surgical environments have been developed in various domains, a major drawback is the lack of interaction between the domain experts (or surgeons) and the virtual environment developers as well as the lack of a structured process model as the foundation of such an environment.

A language termed eEML (the engineering Enterprise Modeling Language), was used to develop this model; in the past, eEML has been used by CINBM researchers to model a range of complex processes. This model captures the key or driving assumptions, information inputs, skill constraints, the intermediate 'attribute' outcomes between various steps or stages of the process in reference as well 
as the crucial performing 'agents' (which can range from the medical personnel involved in the diagnosis and surgery itself to the medical assistive devices which play a key role in the outcome of various steps in this surgical process). This information model can be viewed as a baseline information intensive model that captures the functional relationships among related tasks at various levels of abstraction but also enables the representation of temporal precedence constraints among sub-tasks [26].

The information model was built after interaction with Dr. Pirela-Cruz (who is an experienced micro surgeon) and was developed through interviews, discussions, attending cyber lectures, watching videos, etc. After identifying the core tasks involved in a target micro surgical process, subsequently the other details were identified including the constraints, the information inputs and decision outcomes for tasks and sub tasks in this process. We also captured the temporal precedence constraints for the accomplishment of these tasks. By emphasizing a process level of details, our approach has sought to satisfy the functional requirements of micro surgery.

Figure 12 illustrates the importance of the role of the information models. The information models can be viewed as an outcome of understanding the complex surgical process. Subsequently, it becomes the foundation to create the Virtual Surgical Environment. In Phase I, we have used process models as a basis to understand the process

of suturing in the virtual environment. In Phase II, we propose to automate this process for various other micro surgical activities.

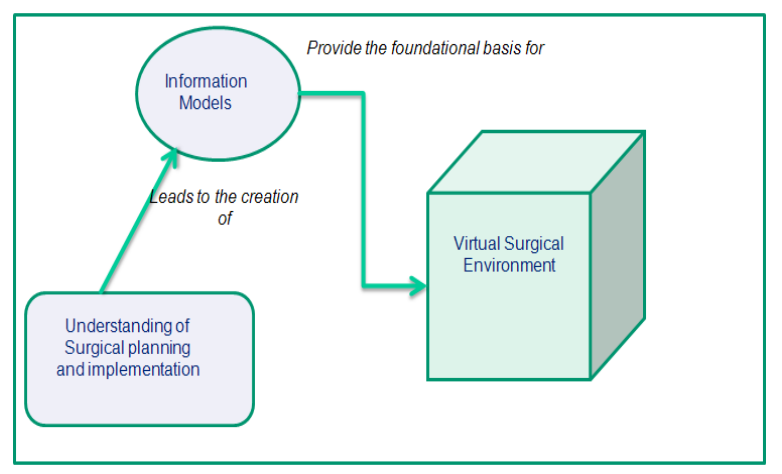

Figure 12: Using Information Models to understand micro surgical processes

As the detailed information model is lengthy, for the purposes of brevity, we will only highlight the top level views of this model. The surgical process can be viewed as set of core tasks and sub tasks (see E-0 in figure 16) along with associated performing entities (called performing agents PA's) and outcomes (called decision objects DO's).

Constraints, information and physical objects can be modeled as Influencing Criteria (IC's). A core task such as E-0 (in figure 8) can be decomposed into a corresponding set of sub tasks E1...En. The surgical process can then be modeled and studied as a collection of tasks with a corresponding set of relationships. By developing a map from this information model to the simulation environment, the activities (which are simulated within the virtual environment) can be modified as necessary. By using relevant temporal constraints (such as 'AND' and 'OR'), the modeling of synchronous and asynchronous tasks can also be modeled explicitly.

In an eEML model there are four categories of information attributes: the Influencing Criteria (IC), the Associated Performing Agents (APA), the Decision Objects (DO) and the Task Effectors (TE). Using these attributes, a target set of activities can be modeled, studied and analyzed at various levels of abstraction. Influencing Criteria can be categorized as information inputs (II) and constraints (CO), which directly impact the accomplishment of the target activity (being modeled). Constraints can be viewed as controlling factors influencing the process being modeled. The information inputs are the information attributes which are required to accomplish the target process being modeled.

The associated Performing Agents (PAs) refer to the software, personnel and/ or machines/tools agents, which perform the identified tasks. When modeling a given surgical process, PAs will typically refer to the surgeon (who is performing a given surgery as well as others assisting in the surgery), the various instruments used by the surgeon such a needle holder (fig. 13), needle (fig. 14), etc.. When the Scenario Manager extracts such information, a corresponding scenario is simulated using such details. When a different type of physical resources or instruments are involved, by changing the corresponding information attributes or entities in a eEML model, the simulation scenario can quickly be modified. Using such an approach is beneficial in the long term as it will allow surgeons to explore the feasibility of using various instruments rapidly and then analyzing the associated surgical 'scenarios' through the corresponding simulations in the virtual environment.

Decision Objects (DO) can be grouped under information and physical objects, which refer to the information or physical outcomes (respectively) of activities performed. In a surgical process, the outcomes of each activity becomes crucial and serves as the basis for the subsequent activity. In eEML, the change is modeled at the lower level as a change in the status of an existing scenario. For example, when a specific task such as clamping a blood vessel is completed, this is noted as a state change described by a noun such as 'clamped blood vessel'. The state change is noted as an entity under 'Physical Object' to highlight the fact a physical attribute (corresponding to the various entities involved in the relevant activity being modeled) has undergone a change. By storing and noting additional information (which can vary depending on the nature of the surgical process or sub-process) such as new information or feedback 
information, a detailed model of complex surgical processes can be built.

The end effectors indicate flow of task accomplishment in either a synchronous or asynchronous manner (synchronous accomplishment is represented using a symbol ' $\&$ ' and asynchronous tasks can be represented using ' $V$ '). Logical 'AND' and 'OR' relationships can also be represented. In general, a surgical process is composed of a series of sequential activities. However, when a team of surgeons are involved, concurrent activities can be undertaken as part of the overall process. eEML supports the modeling of such activities through these logical 'AND' as well as 'OR' functions. A Synchronous symbol used at a junction (where three preceding activities have to be completed) can represent a constraint condition that until the preceding activities are completed, a subsequent target activity will not be initiated.

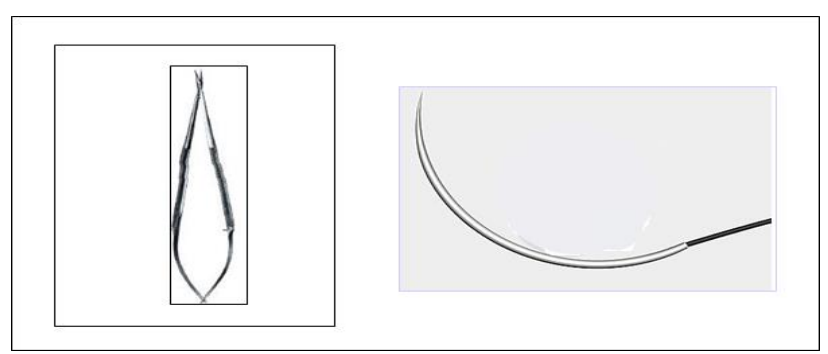

This information model was developed after closely interacting with a micro surgeon Dr. Pirela-Cruz; use of such an information model has been critically lacking in other virtual surgical research initiatives. Figures 19 are images of some of the various tools during micro surgery. The associated CAD models were created in Solidworks and used to develop the virtual environment discussed in this paper.
Figure 13: A needle holder (left) and Figure 14: close-up view of a micro surgery needle (right)

A list of the major attributes used in eEML is shown in Figure 15 along with their abbreviations (which appear in the eEML diagrams). Consider the eEML diagram for the suturing process as shown in figure 15. This diagram, which represents the highest level of abstraction, illustrates the general layout and use of attributes in eEML (for suturing process in this case). The primary, secondary and tertiary importance of the various attributes is indicated using ' $\mathrm{P}$ ', ' $\mathrm{S}$ ' and ' $\mathrm{T}$ ' symbols. This is determined by the modeler based on their perception of the level of importance of a specific attribute.

A modeled (target) activity E-0 can be decomposed into a set of related activities E-1, E-2, etc. In general, after the context, modeling perspective and objective have been formalized; the focus unit level is created. At this E-0 level, the various ICs, APAs, and DOs are identified as well as prioritized (as primary, secondary and tertiary); the temporal relationships among these activities are also captured using appropriate junctions. Subsequently, the tasks that comprise the focus unit are identified in the decomposition and the process is repeated.

\section{AN EXAMPLE INFORMATION MODEL}

For purposes of brevity, the discussion in this section is limited to the process of performing a suture on blood vessels. The detailed information models developed includes planning and surgical activities for a variety of procedures using an array of surgical options. 


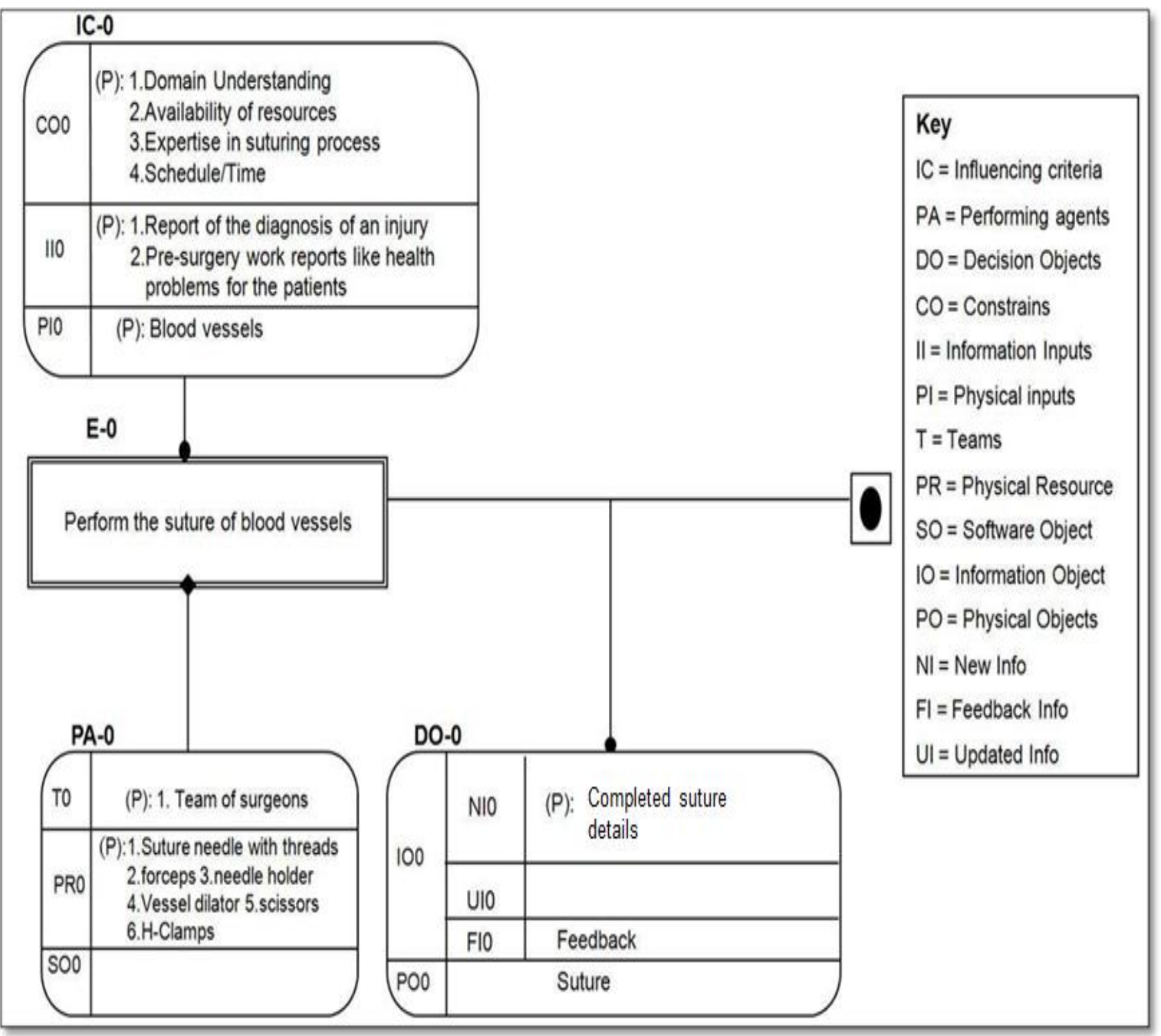

Fig 15: (E-0) Top Level view for eEML Model 
Consider the top level process (E-0) 'Perform the suture of Blood Vessel'. This is the main process being modeled (viz. suturing a blood vessel). At the E-0 level, the influencing criteria shown in figure 9 include constraints such as domain understanding, schedule, expertise in suturing process, availability of resources etc. This model not only provides the inputs required before surgery but also identifies key decisions in the surgical process. The information inputs provided are reports of the diagnosis of the injury and any preoperative medical problems associated with the patient. The key decision outcomes include the successful completion of the surgery (which is a suture, indicated in the physical object category in figure 15). Figure 16 and 17 show the decompositions of E-1 through E-6. Only these decompositions at the top level are shown for brevity. The key objects are the Physical Object (PO) entities in theses diagrams. They indicate the status changes as the micro surgery task progresses. For example, clamped blood vessel, positioned needle, vessel with inserted needle, complete knot and completed suture are the key status changes in the overall progress of a successful surgical process. An overview of the 6 activities comprising E-0 follows.

\section{E-1: Prepare the vessel}

In this pre-surgery stage, the surgeon lifts the vessel and cleans the tissue and blood within the vicinity of the blood vessel with the help of 0.025 markcaina solution, the scissors and scalpel. The surgeon then expands the vessel with the help of a vessel dilator and clears the lumen. The key constraints in this stage are availability of resources and expertise in suturing process. The information input for this process is diagnosis of the injury (report). The key performing agents are Team of surgeons and the physical resources are the tools used viz. Forceps, Scissors and scalpels and the markcaina solution used for cleaning the vessel.

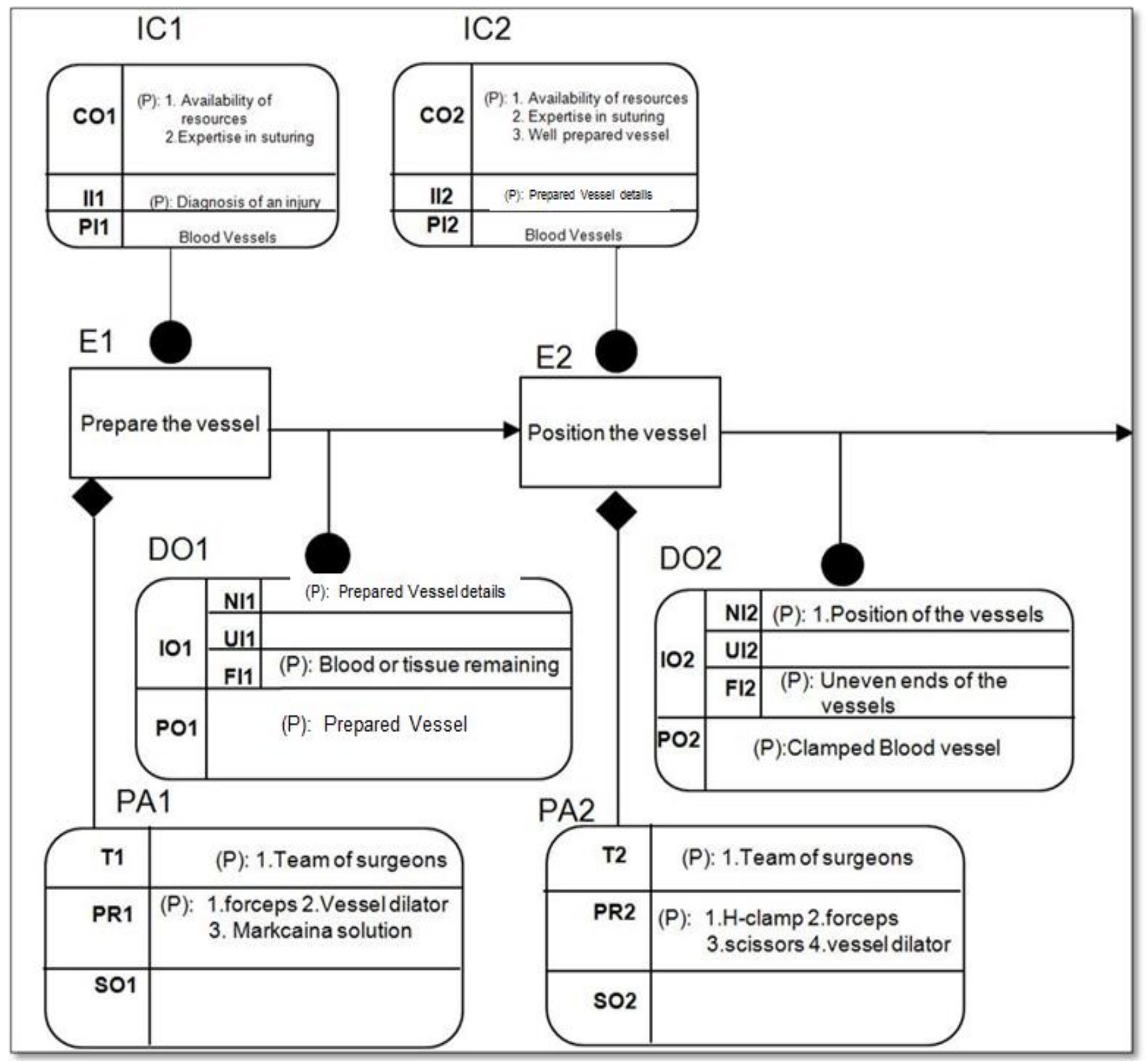

Fig 16: Decomposition of E0 level showing E-1 and E-2 
E2: Position the blood vessel: In this step, the surgeon positions the cleaned vessel correctly. He/she makes both the ends of the vessel of the same size to remove the irregularity in their size so that the suturing can be performed with precision. The surgeon positions the $\mathrm{H}$ clamps on the vessel in such a way that the vessels are neither too loose nor held too firm.

E3: Position the needle: The surgeon then positions the suture needle with a curved needle holder. He/she surgeon makes the needle point in the right direction/angle and avoids the downward pointing of the needle to prevent the back clamping of the blood vessel.

E4: Pass the needle through the vessel: The surgeon averts the tissue edge to provide distortion and to make the needle pass at right angles through the tissue. Using a left hand forceps on the topside of the tissue, the surgeon makes the needle follow its own curvature. This is performed to avoid the back clamping of the vessel. The surgeon avoids the 'through stitch' and pulls the needle through in one straight movement and in the process pulls the suture through.

E5: Perform the knotting procedure: This is the actual suturing process. The surgeon ties the surgical knot in which she/he picks up the suture with a left hand forceps and makes a loop on the tip of the right hand forceps. The short end of suture is picked up with the right hand close to the loop length. The surgeon makes a single loop and then completes the first half knot. The surgeon then turns the needle holder forceps and pulls the loop, tightening the knot. The longer segment of the thread with forceps is held, pulling the loop and tightening the knot.

E6: Perform Patency Tests: This is the post-surgery step in which the surgeon removes the H-Clamps, cleans the vessels and the area in the vicinity and performs the patency tests to check whether the vessel is open and blood flows through it.

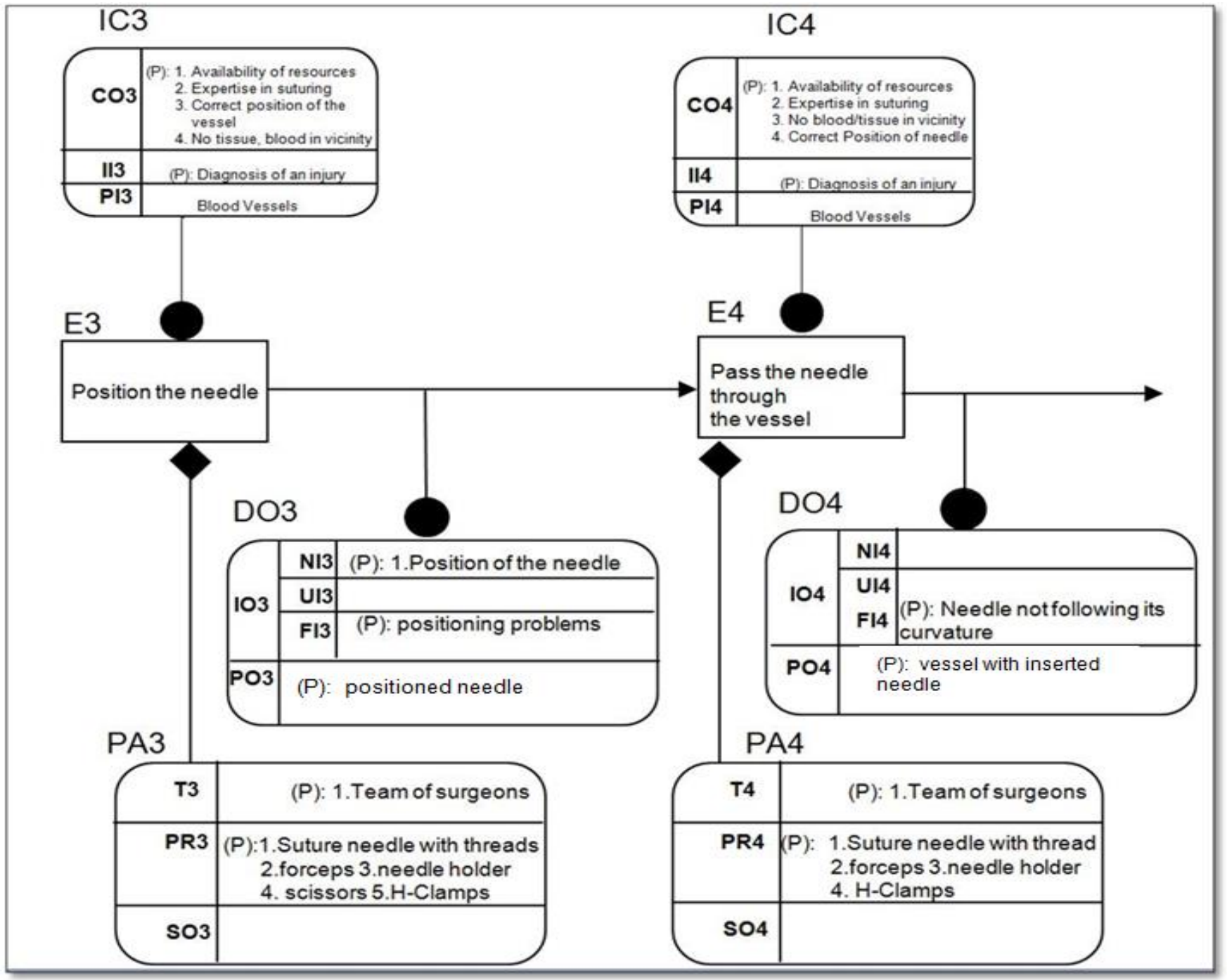




\section{DISCUSSION AND CONCLUSION}

Organic objects (or entities) such as the blood vessels (arteries, etc.) can be created by using sophisticated mesh models and then imported into a software tool such as Chai3D. Chai 3D maintains a scene graph type of representations which allows fundamental objects including world (graphic universal object), camera (view), and light source (visibility) with appropriate arguments.

Our literature review has indicated that while various other virtual environments for surgery have been designed and reported in the literature, none of the previous approaches have adopted the use of an information model to understand surgical processes $[25,26]$. Our approach emphasizes the need to create information models as a basis for designing and building virtual environments (figure 12). This paper highlighted the innovative use of information models in first developing a formal understanding of the complex activities in micro surgical processes. This understanding is important and lays a robust foundation to the design of virtual surgical environments. In our approach, a language called eEML (engineering Enterprise Modeling Language) was used to build this information model.

It should also be noted that other reported approaches have not explored the use of information models to propel the various simulation activities in a virtual environment. In our approach, we emphasize the use of information models (or information intensive process models), which can be used to propel corresponding simulation activities in a virtual environment.

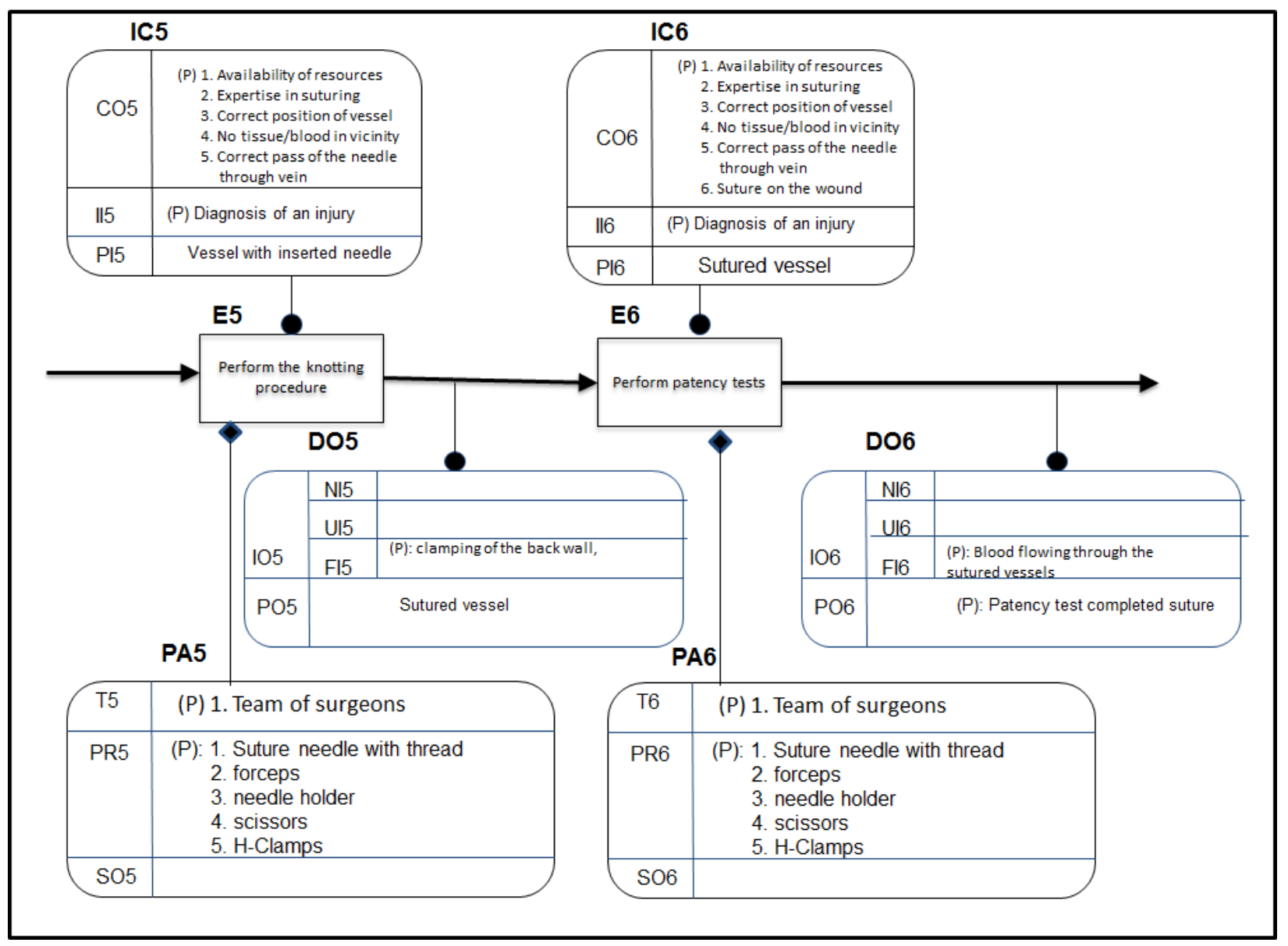

Fig 18: Decomposition of E0 level (contd.) 
Using such an approach enables a target simulation scenario to be modified through this information model. This information model driven simulation approach is unique (first of its kind) and is a core part of our methodology to develop virtual environments for surgical processes. While the eEML model provides a great level of detail as well as modeling flexibility, it is important to note that this information model has to be built with an attention to detail. The modeler needs to interact closely with the process experts (surgeons) to build an information rich model which captures the complex relationships within a target process. Subsequently, the details in such a model needs to be verified to ensure correctness $[25,26]$.

Potential plans for the future include incorporating the haptic interface to the virtual surgical environment. Additional avatar assisted training modules (with voice cues) will also help in providing a more effective and user friendly training experience.

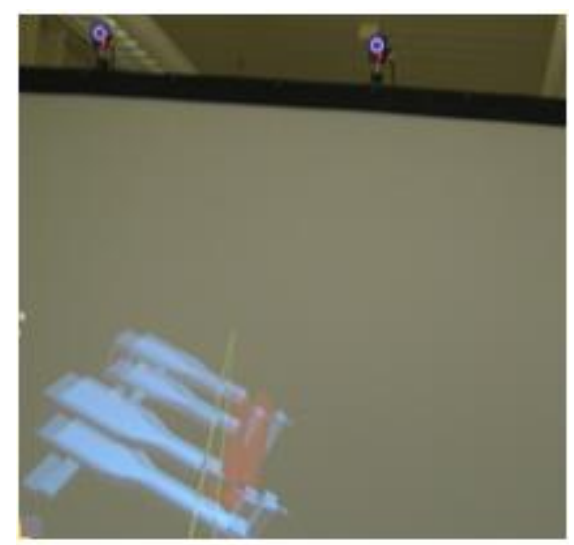

Figure 19: View of the virtual environment on the Virtual Reality PowerWall

\section{REFERENCES}

[1] Van der Meijden OA; Schijven MP ."The value of haptic feedback in conventional and robot-assisted minimal invasive surgery and virtual reality training: a current review." Surgical Endoscopy (1432-2218) 2009 Jun. Vol.23,Iss.6;p.1180-90

[2] Christian R Larsen; Jette L Soerensen; Teodor P Grantcharov; Torur Dalsgaard; Lars Schouenborg; Christian Ottosen; Torben V Schroeder; Bent S Ottesen. "Effect of virtual reality training on laparoscopic surgery: randomized controlled trial". British Medical Journal

[3] O'Leary, SJ."Validation of a Networked Virtual Reality Simulation of Temporal Bone Surgery". LARYNGOSCOPE. JUN 2008. Vol.118,Iss.6;p.1040-1046

[4] Cecil, J., Use of EML in design and development of software systems in manufacturing. Technical Report. Virtual Enterprise Engineering Laboratory (VEEL). Industrial Engineering department, NMSU. Las Cruces, New Mexico, 2002.

[5] Yaacoub, Fadi; Hamam, Yskandar; Abche, Antoine."A virtual reality simulator for training wrist arthroscopic surgery". HEALTHINF 2009 - Proceedings of the 2nd International Conference on Health Informatics 2008. p.74-81

[6] Wang, P.; Becker, A.A.; Jones, I.A.; Glover, A.T.; Benford, S.D.; Greenhalgh, C.M.; Vloeberghs, M.; Wang, P."Virtual reality simulation of surgery with haptic feedback based on the boundary element method." Computers \& Structures (0045-7949) 4/1/2007. Vol.85,Iss.7/8;p.331-339

[7] Wang, P.; Becker, A.A.; Jones, I.A.; Glover, A.T.; Benford, S.D.; Greenhalgh, C.M.; Vloeberghs, M.; Wang, P.”A virtual reality surgery simulation of cutting and retraction in neurosurgery with force-feedback." Computer Methods \& Programs in Biomedicine (0169-2607) 10/1/2006. Vol.84,Iss.1;p.11-18

[8] Soler, L.; Nicolau, S.; Schmid, J.; Koehl, C.; Marescaux, J. Pennec, X.; Ayache, N."Virtual reality and augmented reality in digestive surgery Mixed and Augmented Reality". 2004. ISMAR 2004. Third IEEE and ACM International Symposium on (07695-2191-6) 2-5 Nov. 2004. p.278

[9] Pheng-Ann Heng; Chun-Yiu Cheng; Tien-Tsin Wong; Yangsheng Xu; Yim-Pan Chui; Kai-Ming Chan; Shiu-Kit Tso; Pheng-Ann Heng. "A Virtual-Reality Training System for Knee Arthroscopic Surgery."IEEE Transactions on Information Technology in Biomedicine (1089-7771)6/1/2004. Vol.8,Iss.2;p.217-227

[10] Guicai Zhang; Shushang Zhao; Yangsheng Xu;"'A virtual reality based arthroscopic surgery simulator".Robotics, Intelligent Systems and Signal Processing, 2003. Proceedings. 2003 IEEE International Conference on (0-7803-7925-X) 8-13 Oct. 2003 Vol.1;p.272

[11] Ming-Dar Tsai; Ming-Shium Hsieh; Shyan-Bin Jou."simulator". Computers in Biology and Medicine (0010-4825) Sept. 2001 Vol.31,Iss.5;p.333-51

[12] Xia, James; Ip, Horace H.S.; Samman, Nabil; Wong, Helena T.F.; Gateno, Jaime; Dongfeng Wang; Yeung, Richie W.K.; Kot, Christy S.B.; Tideman, Henk; Xia, James.”Three-Dimensional Virtual-Reality Surgical Planning and Soft-Tissue Prediction for Orthognathic Surgery." IEEE Transactions on Information Technology in Biomedicine (1089-7771)

[13] Kevin Montgomery et all "SPRING: A General Framework for Collaborative, Real time surgical simulation." National Biocomputation Center, Stanford, CA 94305, USA

[14] Syed Haque, Shankar Srinivasan." A Meta-Analysis of Training Effectiveness of Virtual Reality Surgical Simulators." IEEE Transaction on Information Technology in Biomedicine, Vol.10, No.1, Jan 2006

[15] Bernhard Reitenger, Alexander Bornik, Reinhard Beichel, Dieter Schmalstieg." Liver Surgery Planning Using Virtual Reality."Graz University of Technology. Published by IEEE Computer System

[16] Cristian Luciano, P Banerjee, Thomas DeFanti." Haptics-based virtual reality periodontal training simulator." University of Illinois at Chicago, Chicago, USA, DOI 10.1007/s/10055-0090112-7

[17] N.E Seymour." Virtual Reality in General Surgical Training". Division of General Surgery, Bay state Medical Center, Springfield, Massachusetts, U.S.A.Eur surg (2005) 37/5: 298 303; DOI 10.1007/s10353-005-0184-x

[18] Paul A. Kay; Richard A. Robb; Robert P. Myers; Bernie F. King."Creation and validation of patient specific anatomical models for prostate surgery planning using virtual reality." Visualization in Biomedical Computing

[19] Peng, Xiaobo; Chi, Xiaoyi; Ochoa, Jorge A.; Leu, Ming C.’Bone surgery simulation with virtual reality."Proceedings of the ASME Design Engineering Technical Conference 2003. Vol.1 B;p.11051113

[20] Baumann, Roger; Clavel, Reymond."Simulation". Proceedings IEEE International Conference on Robotics and Automation (1050-4729)1998. Vol.1;p.381-386

[21] Machado LS; Moraes RM; Souza DF; Souza LC; Cunha IL."A framework for development of virtual reality-based training simulators."Studies In Health Technology And Informatics (0926-9630) 2009. Vol.142;p.174-6

[22] Bayona, Sofía; Fernández-Arroyo, José Manuel; Bayona, Pilar; Pastor, Luis."A new assessment methodology for virtual reality surgical simulators." Computer Animation and Virtual Worlds (1546-4261) January-February 2009. Vol.20,Iss.1;p.39-52

[23] Frank, A.O.; Twombly, I.A.; Barth, T.J.; Smith, J.D. "Finite element methods for real-time haptic feedback of soft-tissue models in virtual reality simulators."(0769509487)2001. p.25763, xxii+326 pp

[24] Jalote-Parmar, A. and P. Badke-Schaub (2008). "Workflow Integration Matrix: A Framework to Support the Development of Surgical Information Systems." Design Studies 29 (4): 338-368

[25] Cecil, J., Cruz, M., An Information Modeling Approach to support process design activities in Micro Surgery, presentation at the 2010 Industrial Engineering Research Conference, Cancun, Mexico, June 5 -9, 2010 
[26] Cecil, J., Cruz, M., Virtual Environments for Bio Surgery, presentation at the 2010 Tools and Methods in Competitive Engineering, Ancona, Italy, April 12-15, 2010.

[27] Cecil, J., Representation Issues of Physiological Attributes in Micro Surgery, Project Report, Center for Information Based Manufacturing, Fall 2009, Oklahoma State University, Stillwater, $\mathrm{OK}$.

[28] Berg, D., et al, Virtual Reality Simulators for Dermatologic Surgery: Measuring Their Validity as a Teaching Tool, Dermatologic Surgery, Volume 27 Issue 4 Page 370-374, April 2001.

[29] Nakao M, Oyama H, Komori M, Matsuda T, Sakaguchi G, Komeda M, Takahashi T., Haptic reproduction and interactive visualization of a beating heart for cardiovascular surgery simulation, International Journal of Medical Information, 2002 Dec;Vol. 68(1-3), pp. 155-63.

[30] D. Tzovaras, G. Nikolakis, G. Fergadis, S. Malasiotis, and M. Stavrakis, "Design and Implementation of Haptic Virtual Environments for the Training of the Visually Impaired," IEEE Transactions on Neural Systems and Rehabilitation Engineering, vol. 12, pp. 266-278, 2004.

[31] J. P. Fritz and K. E. Barner, "Design of a haptic data visualization system for people with visual impairments," IEEE Transactions on Rehabilitation Engineering, vol. 7, pp. 372- 384, 1997.

[32] Ahlberg, Gunnar; Enochsson, Lars; Gallagher, Antony; Hedman, Leif; Hogman, Christian; McClusky III, David; Ramel, Stig; Smith, Daniel; Arvidsson, Dag. "Proficiency-based virtual reality training significantly reduces the error rate for residents during their first 10 laparoscopic cholecystectomies." The American Journal of Surgery Vol. 193 (2007); p. 797-804

[33] Thomas Stadie, Axel; Alfons Kockro, Ralf; Reisch, Robert; Tropine, Andrei; Boor, Stephen; Stoeter, Peter; Perneczky, Axel. "Virtual reality system for planning minimally invasive neurosurgery." Journal of Neurosurgery(2008). Vol 108. p-382394.

[34] Seymour, Neil. "VR to OR: A Review of the Evidence that Virtual Reality Simulation Improves Operating Room Performance." World Journal of Surgery (2008) Vol 32. p-182188.

[35] Cecil, J., A Functional Model of Fixture Design to aid in the design and development of automated Fixture Design systems', Journal of Manufacturing Systems, August 2002, Vol. 21, No.1, pp.58-72.

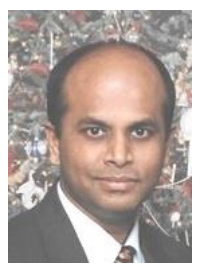

Dr. J. Cecil is an Associate Professor in Industrial Engineering and directs the Center for Information Centric Engineering CICE) at Oklahoma State University. His interests are primarily in the area of Information Centric Engineering (ICE) with an emphasis on 3 core facets: (a) modeling, (b) simulation / visualization and (c) exchange of information. Current research projects explore ICE issues in emerging process domains including micro assembly, space systems and bio medical engineering His research thrusts include the design of information intensive modeling approaches to facilitate simulation, creation of Virtual Reality based simulation environments for engineering and scientific applications, and the design of cyber physical frameworks for collaborative engineering and educational contexts.

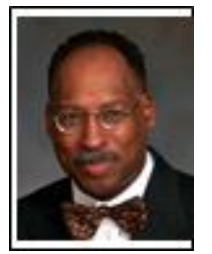

Miguel A. Pirela-Cruz, MD, FACS is a Professor and Chairman of the Department of Orthopaedic Surgery, Paul L. Foster School of Medicine at the Texas Tech Medical Center Health Science Center in El Paso, Texas. Dr. Cruz is a practicing Orthopaedic and Microsurgeon with patient care, teaching and research responsibilities. Over the past few years, Dr. Cecil and Dr. Cruz have been exploring the design of virtual environments to train young surgeons and help them acquire the necessary skills to perform microsurgery. His past projects included the developments of new surgical techniques to treat congenital, traumatic and acquired conditions that affect the extremities. His current clinical research involves reconstruction of Axillary Nerve injuries by transfer of the Thorocodorsal Nerve. He has developed several orthopaedic implants that are currently used today. 